\title{
Energy Efficiency Oriented Design Method of Power Management Strategy for Range-Extended Electric Vehicles
}

\author{
Jiuyu Du, Jingfu Chen, Mingming Gao, and Jia Wang \\ State Key Laboratory of Automotive Safety and Energy, Tsinghua University, Beijing 100084, China \\ Correspondence should be addressed to Jiuyu Du; dujiuyu@tsinghua.edu.cn
}

Received 9 March 2016; Revised 3 June 2016; Accepted 6 June 2016

Academic Editor: Muhammad N. Akram

Copyright (C) 2016 Jiuyu Du et al. This is an open access article distributed under the Creative Commons Attribution License, which permits unrestricted use, distribution, and reproduction in any medium, provided the original work is properly cited.

\begin{abstract}
The energy efficiency of the range-extended electric bus (REEB) developed by Tsinghua University must be improved; currently, the energy management strategy is a charge-deplete-charge-sustain (CDCS) strategy, which exhibits low energy efficiency on the demonstration model. To improve the energy efficiency and reduce the operating cost, a rule-based control strategy derived from the dynamic programming (DP) strategy is obtained for the Chinese urban bus driving cycle (CUBDC). This rule is extracted by the power-split-ratio (PSR) from the simulation results of the dynamic powertrain model using the DP strategy. By establishing the REEB dynamic models in Matlab/Simulink, the control rule can be achieved, and the power characteristic of powertrain, energy efficiency, operating cost, and computing time are analyzed. The simulation results show that the performance of the rule-based strategy presented in this paper is similar to that of the DP strategy. The energy efficiency can be improved greatly compared with that of the CDCS strategy, and the operating cost can also be reduced.
\end{abstract}

\section{Introduction}

Environmental concerns and increasing fuel cost have motivated manufacturers and governments to develop alternative technologies to replace conventional internal combustion engine (ICE) vehicles [1]. Recent works have shown that vehicle electrification can reduce energy waste and minimize fuel consumption [2]. Nevertheless, battery electric vehicles (BEVs) still have a major drawback: energy storage. For massive deployment of BEVs, the problems of driving range, charging time, lifetime, and higher upfront cost must be solved. Typically, a BEV stores energy in batteries that are bulky, heavy, and expensive. Due to this problem, with current battery technology, it is very difficult to make a general purpose BEV that effectively competes with ICE vehicles [3].

A range-extended electric vehicle (REEV) provides a platform to overcome the BEV's drawbacks and reduce fuel consumption and energy waste [4]. REEVs utilize a smaller size power battery than that of battery electric vehicle along with a small displacement range extender; this combination is an optimal solution to the economic and energy storage issues of BEVs. In China, public buses are regarded as the priority concerning development of new energy vehicles (NEVs). A type of range-extended electric bus has been developed by Tsinghua University (THU) and automotive companies and demonstration operations have been performed in cities for several years. Presently, the fuel efficiency of the REEB is too low and urgently must be improved. The energy efficiency is an important criterion to assess the performance of a range-extended electric bus, and the energy management strategy is a key influencing factor on the energy consumption [5]. The present energy management strategy of the THU REEB is charge-depletecharge-sustain (CDCS), which is established by engineering experience; as a result, the energy efficiency still has much room for improvement, and a new control strategy should be developed. The energy management strategy of a hybrid electric powertrain can be classified into instantaneous strategies, global optimized strategies, or rule-based strategies [6]. The key to solving instantaneous optimization problems is a reasonable objective function, such as the equivalent consumption minimization strategy. García et al. [7] and Geng et al. [8] utilized the equivalent fuel consumption 
minimization strategy to analyze the performances of a fuel cell electric vehicle and a plug-in electric vehicle, respectively. Sciarretta et al. [9] and Barsali et al. [10] applied the equivalent fuel consumption minimization strategy on series hybrid electric vehicles and parallel hybrid electric vehicles, respectively. By comparison, the instantaneous optimization is only based on current control step and real-time distribution strategy; as a result, this strategy will affect performance of hybrid powertrain greatly. Thus, a more optimal result can be obtained using global optimization [11]. The DP algorithm is a widely used global optimization method, which is suitable for optimizing the control strategy when the driving cycle is known in advance. Kum et al. [12] and Lin et al. [13] applied the DP strategy on a plug-in electric vehicle and a parallel hybrid electric vehicle, respectively, to achieve better fuel consumption and emissions. However, this type of strategy is difficult to apply on a vehicle due to its heavy computational burden [14]. Rule-based strategies are mainly designed in accordance with engineering experiences and easy to be applied on vehicles. However, the optimal performance is difficult to achieve using rulebased strategies [15]. He et al. [16] presented several rulebased control strategies, such as the voltage control, current control, and brake regeneration control on fuel cell electric bus by simulation. Wu et al. [17] devised a CDCS strategy on the range-extended electric bus in the Harbin bus driving cycle. Gong et al. [18] and Schouten et al. [19] devised a fuzzy logic controller for a parallel hybrid electric bus. Gong et al. [18] developed a neural network control strategy for the energy management system based on the trip model.

The abovementioned optimal control strategies are all global optimal resolution approaches using the DP algorithm, which are difficult to implement on-board because of the computational burden. In contrast, the rule-based control strategy is easy to apply in real-time. This paper combines the advantages of the DP strategy and the rule-based strategy and presents a rule-based strategy derived from the DP strategy to reduce the energy consumption for THU REEB.

\section{Modeling and Simulation}

2.1. Powertrain Configuration and Operational Modes. The schematic diagram of electric powertrain is shown in Figure 1. The range extender acts as on-board generating device consisting of an engine, a generator, and a rectifier. According to the demand of the REEB under different driving cycles, the battery and the range extender provide power to the traction motor to drive vehicle either jointly or separately [17].

2.2. Range Extender Model. The dynamic characteristics of the engine and the generator models are ignored to reduce the computational burden of the DP strategy. Instead, the brake specific fuel consumption (BSFC) map, which is indexed by engine speed and torque, is employed for the fuel consumption calculation, as shown in Figure 2. The same method is adopted for the generator via the efficiency map, as shown in Figure 3. Both maps of the engine and generator are generated using the data from the bench tests.

Given that generator is mechanically coupled to the output shaft of the engine, the generator and engine are at the same working points. The ideal fuel economy curve of the range extender is obtained using the method described in Chen et al. [20]. The fuel consumption map and ideal operation region of the range extender derived from the engine and generator are shown in Figure 4.

2.3. Power Battery Model. Because the RC battery model is too complex for use in the DP process, the battery model is simplified as an equivalent circuit with a voltage source and resistance, as shown in Figure 5. In the simplification, a current response faster than $1 \mathrm{~s}$ is ignored due to the step size of the simplified backward-in-time simulation model. The thermal effect on the battery dynamics is also ignored by assuming the battery temperature does not undergo major changes during vehicle testing. The battery model is as follows [21]:

$$
\begin{aligned}
& \mathrm{SO} C=-\frac{I_{\mathrm{bat}}}{Q_{\mathrm{bat}}}, \\
& \mathrm{SÖC}=-\eta_{\mathrm{SOC}} \frac{U_{\mathrm{OCV}}(\mathrm{SOC})-\sqrt{U_{\mathrm{OCV}}^{2}(\mathrm{SOC})-4\left(R_{\mathrm{int}}(\mathrm{SOC})+R_{t}\right)\left(P_{\mathrm{motor}}(k)-P_{\mathrm{re}}(k)\right)}}{2\left(R_{\mathrm{int}}(\mathrm{SOC})+R_{t}\right) Q_{\mathrm{bat}}},
\end{aligned}
$$

where $I_{\text {bat }}$ is the battery current, $Q_{\text {bat }}$ is the battery capacity, $\eta_{\text {SOC }}$ is the Coulomb efficiency, $U_{\text {OCV }}$ is the open circuit voltage of the battery, and $R_{\text {int }}$ and $R_{t}$ are the internal resistance and thermal resistance, respectively. $R_{\text {int }}$ and $U_{\text {OCV }}$ are functions of the SOC. $P_{\text {motor }}$ and $P_{\text {re }}$ are the output power of the range extender and the input power of the motor, respectively.

The battery charging efficiencies $\eta_{\text {chg }}$ and discharging efficiencies $\eta_{\text {dis }}$ are calculated using [15]

$$
\begin{array}{r}
\eta_{\mathrm{dis}}=\frac{U_{\mathrm{OCV}}-\mathrm{IR}_{\mathrm{dis}}}{U_{\mathrm{OCV}}}=\frac{1}{2}\left(1+\sqrt{1-\frac{4 R_{\mathrm{dis}} P_{\mathrm{bat}}}{U_{\mathrm{OCV}}^{2}}}\right), \\
\eta_{\mathrm{chg}}=\frac{U_{\mathrm{OCV}}}{U_{\mathrm{OCV}}-\mathrm{IR}_{\mathrm{chg}}}=\frac{2}{\left(1+\sqrt{1-4 R_{\mathrm{chg}} P_{\mathrm{bat}} / U_{\mathrm{OCV}}^{2}}\right)}, \\
P_{\text {motor }}(k)-P_{\mathrm{re}}(k)<0,
\end{array}
$$




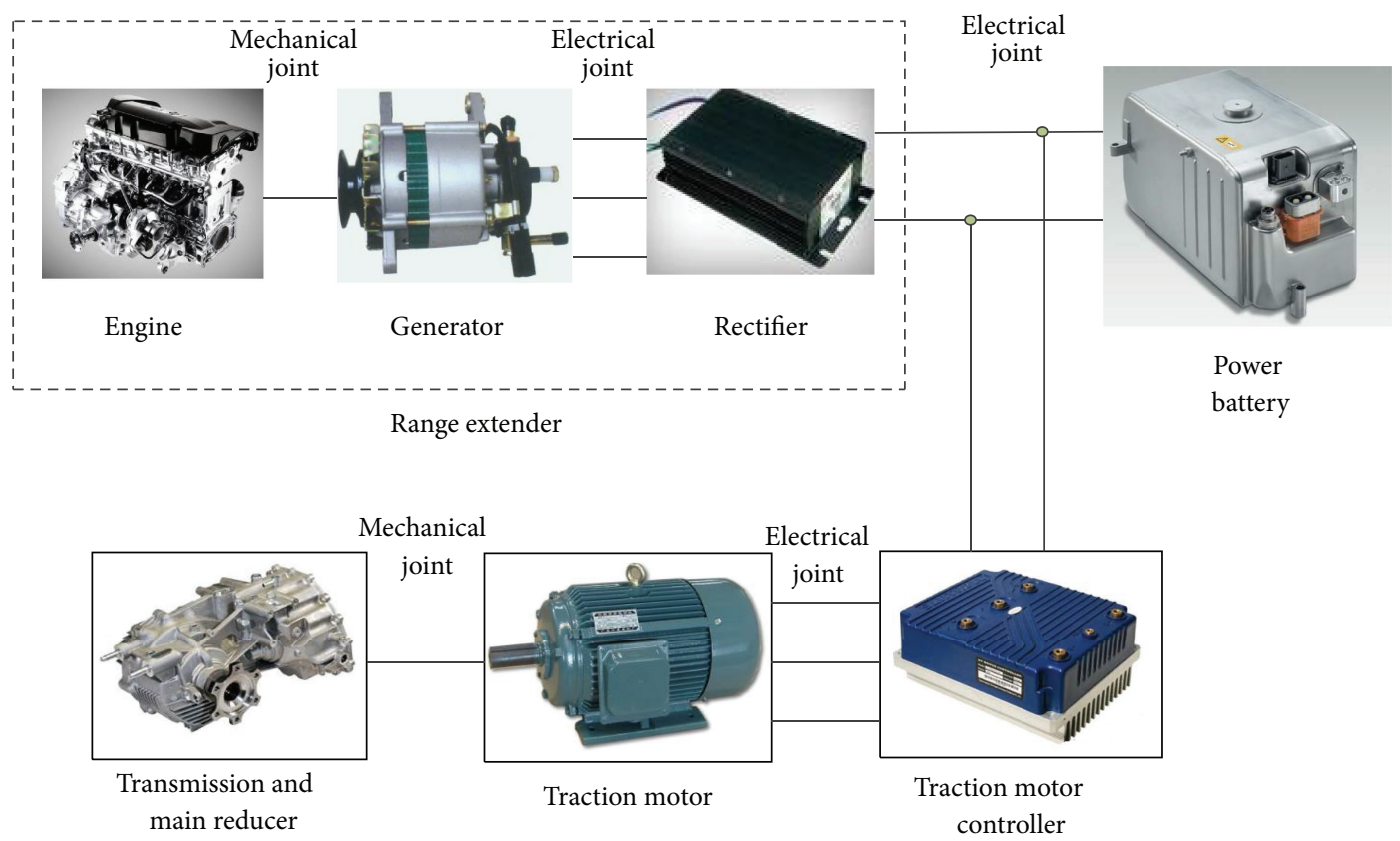

FIGURE 1: Electric powertrain configuration of the THU REEB.

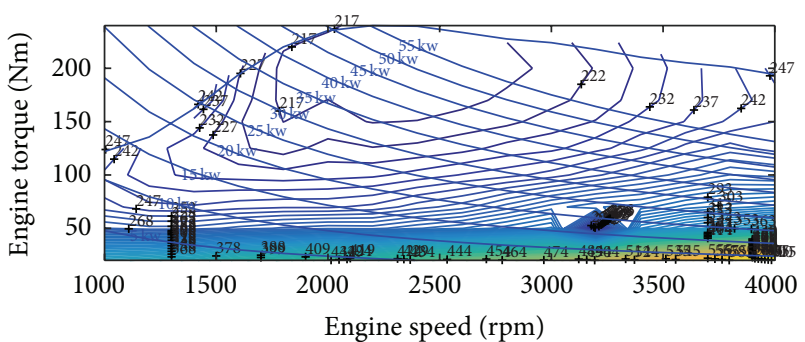

FIGURE 2: Engine BSFC map.

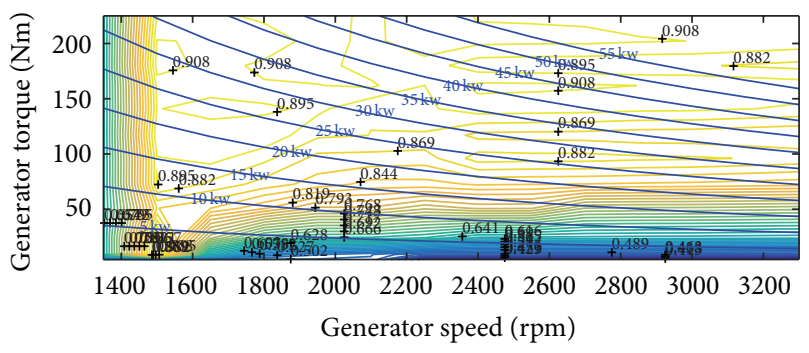

Figure 3: Generator efficiency map.

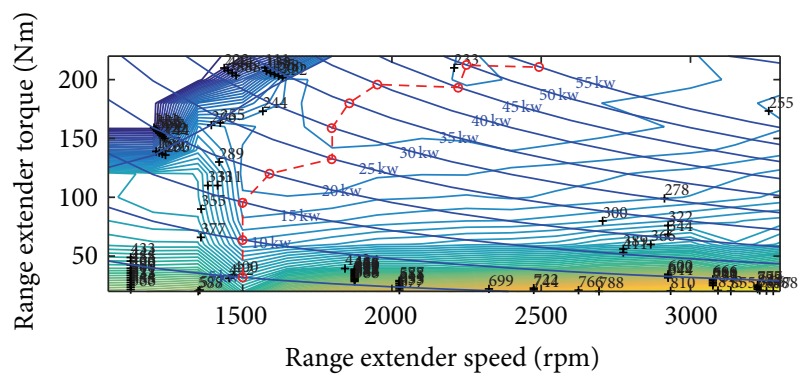

FIGURE 4: Range extender fuel consumption map.

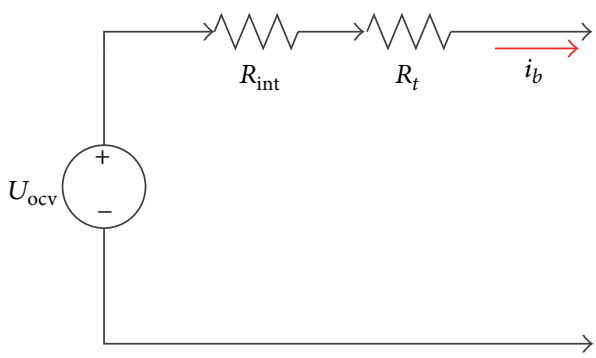

FIGURE 5: Equivalent circuit of simplified battery model.

where $R_{\text {dis }}$ and $R_{\text {chg }}$ are the discharging and charging resistances, respectively.

2.4. Powertrain Modeling. Targeting the fuel consumption, a backward simulation model is established based on the DP strategy. The resistance force of powertrain is expressed as the following state equation:

$$
F_{t}=F_{f}+F_{w}+F_{i}+F_{j},
$$

where $F_{j}$ is the acceleration resistance, $F_{w}$ is the aerodynamic drag force, $F_{f}$ is the rolling resistance, and $F_{i}$ is the slope resistance, and they are determined as follows:

$$
\begin{aligned}
F_{f} & =f\left(m_{c}+m_{p}\right) g \cos \alpha, \\
F_{w} & =\frac{1}{2} C_{d} A \rho v^{2}, \\
F_{i} & =\left(m_{c}+m_{p}\right) g \sin \alpha, \\
F_{j} & =\delta\left(m_{c}+m_{p}\right) \frac{d v}{d t},
\end{aligned}
$$


TABLE 1: Parameters of range-extended electric bus.

\begin{tabular}{|c|c|c|}
\hline \multirow{8}{*}{ Bus } & Curb mass/kg & 13400 \\
\hline & Passenger mass $/ \mathrm{kg}$ & 2760 \\
\hline & Frontal area $/ \mathrm{A} / \mathrm{m}^{2}$ & 7.83 \\
\hline & $C_{d}$ & 0.75 \\
\hline & $f$ & $0.0076+0.00056 v$ \\
\hline & $r / \mathrm{m}$ & 0.512 \\
\hline & $i_{0}$ & 6.2 \\
\hline & $i_{g}$ & 2.18 \\
\hline \multirow{5}{*}{ Motor } & Rated power $/ \mathrm{kW}$ & 100 \\
\hline & Peak power $/ \mathrm{kW}$ & 180 \\
\hline & Maximum torque $/ \mathrm{N} \cdot \mathrm{m}$ & 860 \\
\hline & Maximum speed/r/min & 4500 \\
\hline & Voltage/V & $300 \sim 450$ \\
\hline \multirow{2}{*}{ Engine } & Displacement/L & 1.9 \\
\hline & Maximum output power $/ \mathrm{kW}$ & $82(4000 \mathrm{r} / \mathrm{min})$ \\
\hline \multirow{2}{*}{ Generator and generator controller } & Rated power $/ \mathrm{kW}$ & 50 \\
\hline & Rated torque $/ \mathrm{N} \cdot \mathrm{m}$ & 220 \\
\hline \multirow{2}{*}{ Power battery } & Capacity/Ah & 180 \\
\hline & Voltage/V & $350 \sim 460$ \\
\hline
\end{tabular}

where the parameter $\rho$ is air density, $C_{d}$ is air drag coefficient, $A$ is the frontal area of the vehicle, $f$ is the rolling resistance coefficient, and $\alpha$ is the climbing slope, $v$ is the vehicle speed $(\mathrm{km} / \mathrm{h}), \delta$ is the conversion coefficient of the rotating components mass, $m_{c}$ is the curb mass of range-extended electric bus, and $m_{p}$ is the mass of passengers.

The driving power of powertrain is expressed as follows:

$$
\begin{aligned}
P_{t}= & P_{f}+P_{w}+P_{i}+P_{j}=F_{t} \cdot v, \\
P_{t}= & \frac{\left(m_{c}+m_{p}\right) g f v}{3600}+\frac{\left(m_{c}+m_{p}\right) g \sin \alpha v}{3600}+\frac{C_{d} A v^{3}}{76140} \\
& +\frac{\delta\left(m_{c}+m_{p}\right) v}{3600} \frac{d v}{d t} .
\end{aligned}
$$

The demand power of the motor $P_{\text {motor }}$ is provided by battery and the range extender together or separately as

$$
\begin{aligned}
P_{\text {motor }} & =\frac{P_{t}+3.6 P_{\mathrm{aux}}}{3.6 \eta_{m} \cdot \eta_{\mathrm{ep}} \cdot \eta_{t} \cdot \eta_{\mathrm{fd}}}=P_{\mathrm{re}}+P_{\mathrm{bat}} \\
T_{\text {motor }} & =\frac{F_{t} \cdot r}{i_{g} \cdot i_{0} \cdot \eta_{t} \cdot \eta_{\mathrm{fd}}}
\end{aligned}
$$

where $F_{t}$ is vehicle driving force, $P_{\text {aux }}$ is the demand power of the electrical accessories, $P_{\text {bat }}$ is the battery power, $\eta_{m}$ is the efficiency of the traction motor, $\eta_{t}$ is the efficiency of transmission, $\eta_{\text {ep }}$ is efficiency of the power electronic components, and $\eta_{\mathrm{fd}}$ is the efficiency of the final drive.

The parameters of the range-extended electric bus are shown in Table 1.

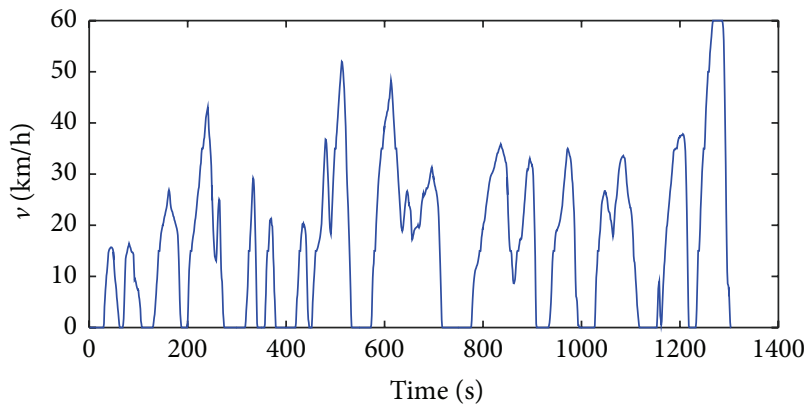

FIGURE 6: Chinese typical urban bus driving cycle.

\section{Driving Cycle}

As the driving cycle is an important factor influencing the energy consumption of electric vehicles, the simulation of the range-extended electric bus is conducted based on the Chinese urban bus driving cycle (CUBDC) shown in Figure 6. Based on the statistical results of the main running lines of the electric buses in typical cities of China, the daily traveled range is approximately $200 \mathrm{~km}$. Therefore, given the large battery capacity of the range-extended electric bus and the one-charge-per-day operation mode, the driving cycle for the simulation is 35 CUBDCs, which spans approximately $200 \mathrm{~km}$.

The DP strategy is suitable for the optimizing energy management system only if the driving cycle is known in advance. The application scope of the preset rule, which is derived from the DP strategy based on CUBDC, should be defined. It is assumed that if the driving cycles are similar to CUDBC, then the abovementioned preset rule by DP can be applied directly. The eigenvalues that can distinguish 
different types of driving cycles should be selected as applicable conditions of the control strategy for different driving cycles. In the research of Montazeri-Gh and Fotouhi [22], the idle rate and the average acceleration of driving cycles can distinguish different types of driving cycles effectively. Therefore, the control strategies in this paper are suitable for the driving cycles with no more than $5 \%$ difference in terms of the idle rate $(39.19 \%)$ and the average acceleration $\left(1.12 \mathrm{~m} / \mathrm{s}^{2}\right)$ of CUBDC.

\section{Energy Management Strategy}

4.1. Overview of the DP Strategy. DP is a mathematical method to design optimal energy management controllers of hybrid powertrain [23], so it serves as benchmark tool with which other energy management strategies are compared.

The state equation for DP strategy in the discrete form is given as follows:

$$
\dot{x}(k+1)=f(x(k), u(k)) .
$$

In the horizon $\left(t_{0}, t_{f}\right)$, the state variables of the rangeextended electric bus powertrain include the battery SOC and the vehicle speed. As the vehicle speed can be determined from the driving cycle, the state variable is $x=$ SOC. According to the optimal objective of minimum equivalent fuel consumption, the output power of range extender is regarded as the control variable, $u=P_{\text {re }}$. The state equation is subject to the following constraints:

$$
\begin{aligned}
& \frac{U_{\text {bus,max }}\left(U_{\mathrm{OCV}}-U_{\text {bus, } \max }\right)}{R_{\text {chg }}} \leq P_{\text {bat }} \\
& \leq \frac{U_{\text {bus,min }}\left(U_{\mathrm{OCV}}-U_{\text {bus,min }}\right)}{R_{\text {dis }}}, \\
& 0 \leq P_{\text {re }} \leq P_{\text {re,max }}, \\
& \mathrm{SOC}_{L} \leq \mathrm{SOC} \leq \mathrm{SOC}_{H}, \\
& T_{m, \text { min }} \leq T_{m}(t) \leq T_{m, \text { max }},
\end{aligned}
$$

where $U_{\text {bus,max }}, U_{\text {bus,min }}$, and $U_{\text {OCV }}$ are maximum voltage, minimum voltage, and open circuit voltage and $R_{\text {chg }}$ and $R_{\text {dis }}$ are charging resistance and discharging resistance of traction battery, respectively. $P_{\text {re,max }}$ is the maximum power of the range extender. $\mathrm{SOC}_{H}$ and $\mathrm{SOC}_{L}$ represent the maximum and minimum values of the SOC, respectively. $T_{m}$ is the traction motor torque; $T_{m, \min }$ and $T_{m \text {, max }}$ represent the maximum torque and the minimum torque of the traction motor, respectively.

The key of the DP strategy is to present a reasonable cost function. In this paper, the electricity consumption is converted to the equivalent fuel consumption, so minimum fuel consumption is regarded as the only optimal objective. The cost function $J$ is given as follows:

$$
J=\sum_{k=0}^{N-1}\left\{C_{\mathrm{re}}(k)+S_{b}(k) \cdot C_{\text {bat }}(k)\right\}
$$

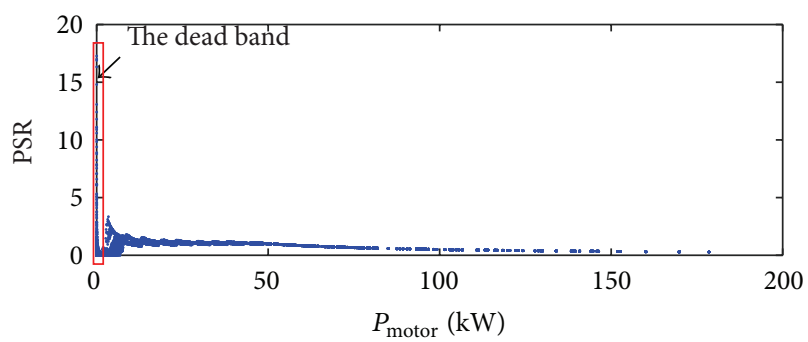

FIGURE 7: Distribution of the PSR points using the DP strategy.

where $C_{\mathrm{re}}$ is the fuel consumption of the range extender, $C_{\mathrm{bat}}$ is the equivalent fuel consumption of the battery, and $S_{b}$ is the coefficient of SOC boundary value. These quantities are calculated as follows:

$$
\begin{aligned}
C_{\mathrm{re}} & =P_{\mathrm{eng}} b_{e} \Delta t, \\
C_{\mathrm{bat}} & =P_{\mathrm{bat}}\left(\eta_{\mathrm{dis}} \eta_{\mathrm{chg}}\right)^{-\operatorname{sgn}\left(P_{\mathrm{bat}}\right)} \frac{C_{\mathrm{re}, \mathrm{avg}}}{P_{\mathrm{re}, \mathrm{avg}}} \Delta t, \\
S_{b} & =1-\frac{2 \mu\left(\mathrm{SOC}-0.5\left(\mathrm{SOC}_{H}-\mathrm{SOC}_{L}\right)\right)}{\left(\mathrm{SOC}_{H}-\mathrm{SOC}_{L}\right)},
\end{aligned}
$$

where $P_{\text {eng }}$ is the output power of the engine, $b_{e}$ is the specific fuel consumption, $C_{\text {re,avg }}$ is the average fuel consumption of the range extender, $P_{\text {re,avg }}$ is the average output power of the range extender, and $\mu$ is the balance coefficient required to maintain the SOC within the reasonable range [24].

4.2. PSR Rule-Based Strategy. To develop the control rule derived from DP strategy, the parameter of power-splitratio (PSR) is proposed indicating the ratio between range extender and motor. PSR is defined as follows:

$$
\operatorname{PSR}=\frac{P_{\text {re }}}{P_{\text {motor }}} .
$$

To minimize the energy consumption using the DP strategy, the range-extended electric bus is modeled and simulated under CUBDC. The PSR points extracted from simulation under DP strategy are shown in Figure 7.

In Figure 7 , when the $P_{\text {motor }}$ is close to $0 \mathrm{~kW}$, the PSR values are distributed dispersedly in the range of 0 to 20 . The consumptions of the fuel and battery energy are taken into consideration simultaneously under the DP strategy to optimize the energy management strategy of the hybrid powertrain. The range extender charges the battery when the $P_{\text {motor }}$ is under low power condition. To find the PSR regular pattern, a dead band was defined as the red box shown in Figure 7. The PSR points in the red rectangle are extracted, and the corresponding range extender power and motor power can be obtained, as shown in Figure 8. It can be seen that the range extender powers in the dead band are no more than $0.2 \mathrm{~kW}$, so they can be ignored for developing the PSR rule-based (PSR-RB) strategy.

The PSR-RB strategy is formulated based on the PSR points outside of the dead band, which can be fitted by the 


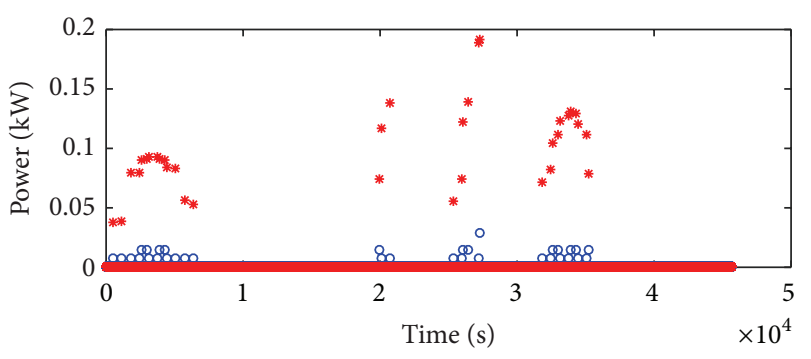

- Range extender power

* Motor power

FIGURE 8: Power feature of the PSR points in the dead band.

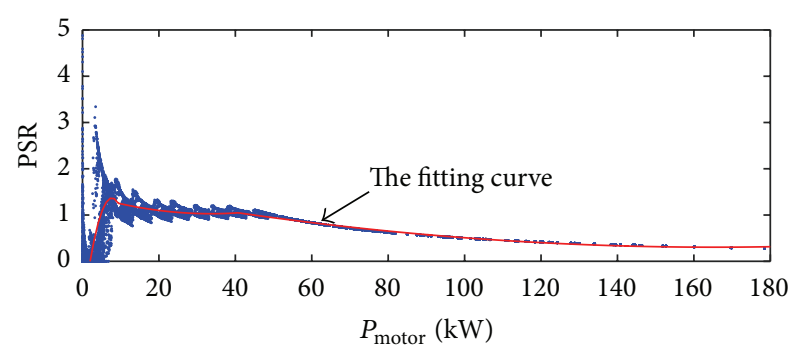

FIGURE 9: Fitting curve according to the PSR points outside of the dead band.

curve shown in Figure 9. The PSR values along red curve are regarded as the power-split rule between the range extender and the battery.

\section{Model Verification}

5.1. Analysis on System Performance. To verify the validity of the aforementioned control strategies, the range-extended electric bus is simulated to analyze the energy efficiency using the DP and PSR-RB strategies on the basis of the CUBDC. Moreover, for comparison to the present strategy of the THU REEB, the CDCS strategy is conducted in the simulation model. When the SOC value decreases to 0.3 , the range extender is forced to charge the battery sometimes in the charge sustaining stage [25]. Variation curves of the battery SOC with the three types of control strategies considered are shown in Figure 10. The PSR-RB strategy is found to be similar to the DP strategy in terms of the SOC variation. However, the computational efficiency is improved significantly for the PSR-RB strategy compared with the DP strategy. The PSR-RB strategy is more responsive, with almost no time delay, and it can be used in real-time as the CDCS strategy to control the range-extended electric bus.

Figure 11 shows the relationship among the motor power, the range extender, and the battery power for the different control strategies. The power features of the PSR-RB strategy are almost in line with those of the DP strategy. Given the driving cycle for the simulation spans approximately $200 \mathrm{~km}$, the battery consumes most of the electricity to supplement the range extender when the demand power of the powertrain exceeds the maximum output power $(50 \mathrm{~kW})$ of the range

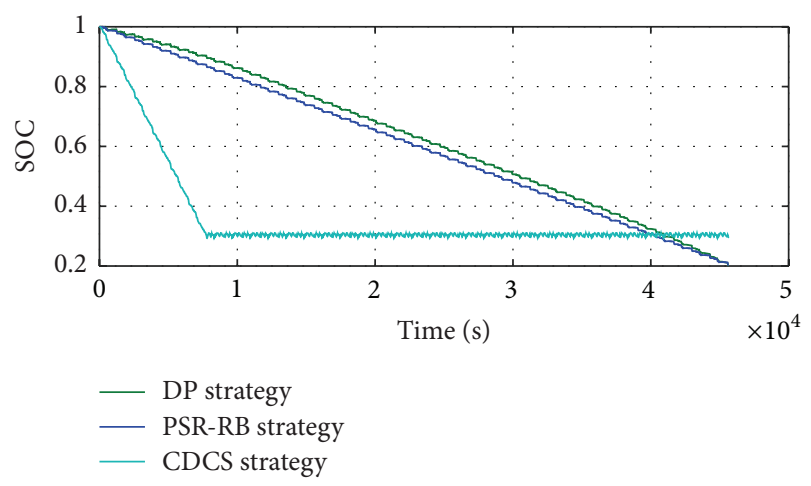

FIGURE 10: SOC decrease curves using the DP, PSR-RB, and CDCS strategies.

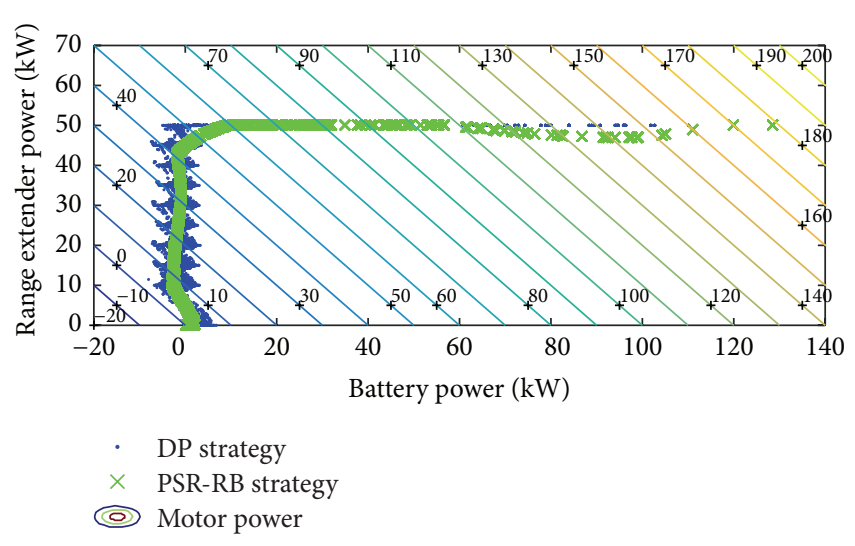

Figure 11: Power features of the components based on the different strategies.

extender. If the demand power of powertrain is lower than $50 \mathrm{~kW}$, then the range extender serves as the main energy source, and the battery charges and discharges over a small range $(-10 \mathrm{~kW}$ to $10 \mathrm{~kW})$ repeatedly to achieve the optimal powertrain efficiency. This process is inherently different compared to the CDCS strategy, which is widely used in engineering practice.

5.2. Energy Consumption and Operating Cost. The electricity and fuel consumption of the REEB powertrain are shown in Table 2. The fuel consumption is improved by $8 \%$ using the DP and PSR-RB strategies compared with the use of the CDCS strategy. For comparison of the energy savings, the fuel and electrical consumptions are converted to MJ-equivalent values [26]. The PSR-RB strategy has a similar energy saving effect as that of the DP strategy, which saves approximately $7 \%$ relative to the CDCS strategy.

The energy flow diagram of the PSR-RB strategy and the CDCS strategy is presented in Figure 12. For a distance traveled of $200 \mathrm{~km}$, the strongest difference between these strategies is the charge energy of the battery, which is from the range extender. Under the CDCS strategy, the range extender produces $507.36 \mathrm{MJ}$ of electrical energy to charge the battery. However, only $38.78 \mathrm{MJ}$ is produced by the range extender in the PSR-RB strategy. In addition, there is a slight 


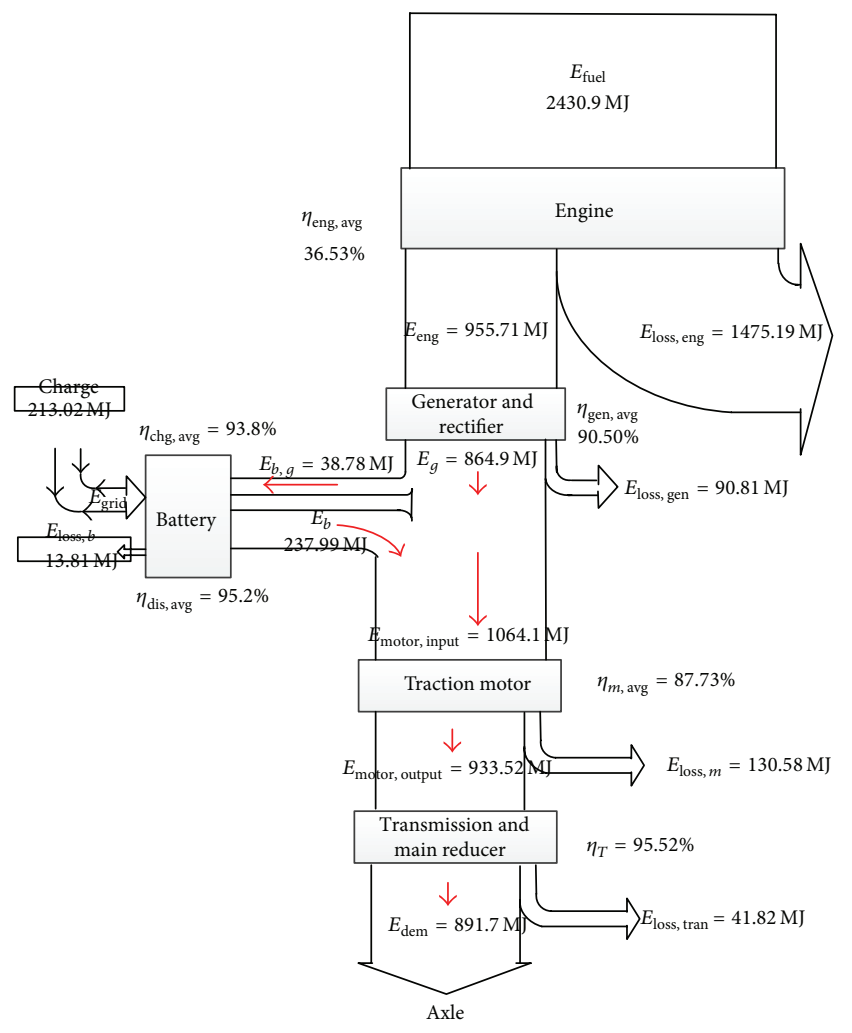

(a) PSR-RB strategy

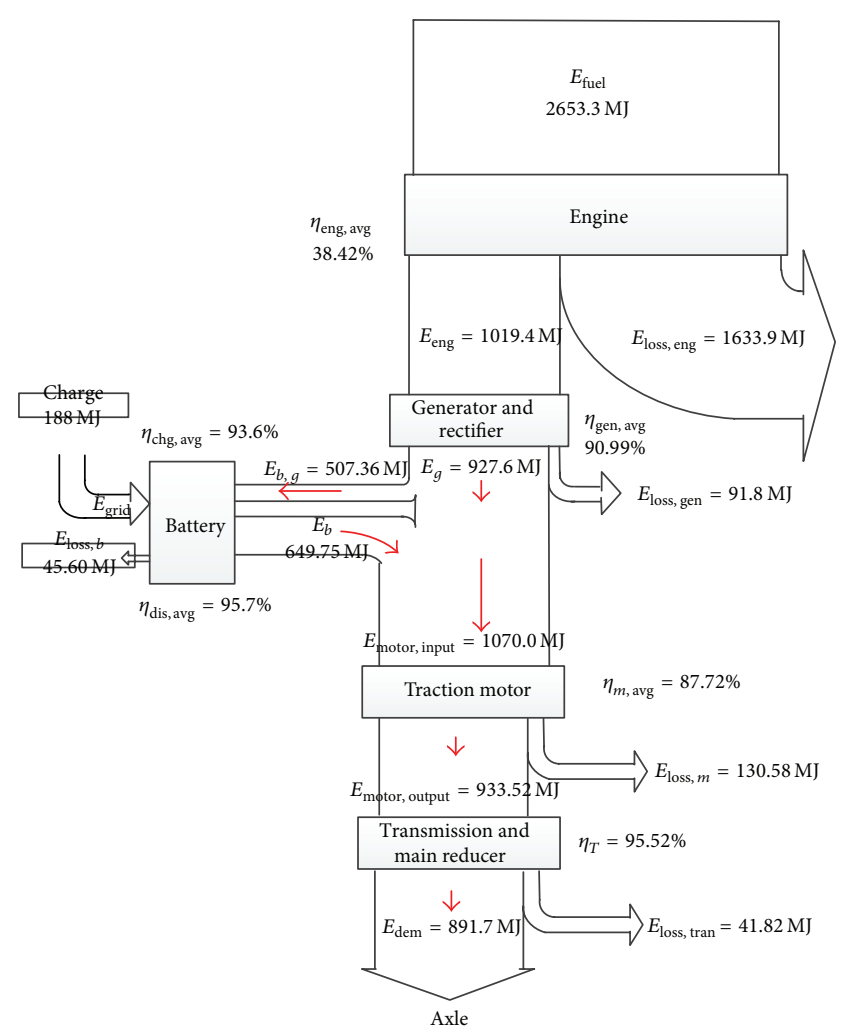

(b) CDCS strategy

FIGURE 12: The energy flow diagram of the REEB powertrain for different strategies.

TABLE 2: Energy consumption comparison.

\begin{tabular}{lccc}
\hline & DP & PSR-RB & CDCS \\
\hline Electricity consumption/kWh & 59.25 & 59.17 & 61.39 \\
Fuel consumption/L & 61.12 & 8.22 \\
Fuel saving rate compared with the CDCS strategy/\% & 8.7 & 7.01 \\
Energy saving rate compared with the CDCS strategy/\% & 7.3 & - \\
Operating cost/RMB per day & 356.92 & 358.38 & 386.83 \\
\hline
\end{tabular}

difference in the engine energy efficiency between the two strategies because the range extender operates at the optimal working points in the CS mode, which improves the engine energy efficiency of entire CDCS mode. Therefore, the PSRRB strategy balances the energy loss between engine energy efficiency and charging the battery via the range extender.

The operating cost is also analyzed. Because the REEB is charged at night, the off-peak electricity price is used for the calculation. The operating cost of the PSR-RB strategy is in agreement with that of the DP strategy; however, compared with the CDCS strategy, the operating cost savings of more than $28 \mathrm{RMB}$ per day are achieved, according to the current electricity and fuel prices in typical cities of China. For the traveled distance $(1,000,000 \mathrm{~km})$ of urban buses during the lifetime of the buses [27], the PSR-RB strategy can achieve more than 140,000 RMB in savings compared with the CDCS strategy.

\section{Conclusion}

To present a new type of energy management strategy that can greatly improve the energy efficiency, reduce operating cost, and meet the real-time range application requirement for the THU REEB, a rule-based control strategy with power splitting characteristic (PSR-RB), which is derived from the DP global control optimal strategy, was investigated. The simulations were conducted to analyze the power characteristic of powertrain, energy efficiency, operating cost, and computing time by different strategies under the China urban bus driving cycle.

The PSR-RB strategy was found to achieve lower fuel and energy consumption compared with those of the CDCS solution. An inherent difference between the proposed strategy and the CDCS strategy was demonstrated in this research. Compared with the CDCS strategy, the fuel savings rate 
and the energy savings rate can reach approximately $8.4 \%$ and $6.9 \%$, respectively, because PSR-RB strategy balances the energy loss between the engine energy efficiency and charging the battery via the range extender to improve the powertrain energy efficiency.

The cost-effectiveness was also demonstrated to be improved by using the PSR-RB strategy. Comparing to the CDCS strategy, the operating cost can be reduced by over $140,000 \mathrm{RMB}$ during the lifetime of the vehicle, according to the current electricity and fuel prices in typical Chinese cities.

Based on the aforementioned simulation results, the range-extended electric vehicle using the PSR-RB strategy exhibits excellent performance, in terms of both energy efficiency and real-time ability; thus, the PSR-RB strategy is a feasible on-board energy management strategy for use in the range-extended electric bus designed by THU.

\section{Competing Interests}

The authors declare that they have no competing interests.

\section{Acknowledgments}

This study is sponsored by new energy vehicles strategic projects of Chinese Academy of Engineering (2015-XZ-3603-03).

\section{References}

[1] N. Denis, M. R. Dubois, R. Dubé, and A. Desrochers, "Blended power management strategy using pattern recognition for a plug-in hybrid electric vehicle," International Journal of Intelligent Transportation Systems Research, vol. 14, no. 2, pp. 101-114, 2016.

[2] M. Honarmand, A. Zakariazadeh, and S. Jadid, "Optimal scheduling of electric vehicles in an intelligent parking lot considering vehicle-to-grid concept and battery condition," Energy, vol. 65, pp. 572-579, 2014.

[3] J. P. Trovão, P. G. Pereirinha, H. M. Jorge, and C. H. Antunes, "A multi-level energy management system for multi-source electric vehicles-an integrated rule-based meta-heuristic approach," Applied Energy, vol. 105, pp. 304-318, 2013.

[4] H. Khayyam and A. Bab-Hadiashar, "Adaptive intelligent energy management system of plug-in hybrid electric vehicle," Energy, vol. 69, pp. 319-335, 2014.

[5] M. Cipek, M. Čorić, B. Škugor, J. Kasać, and J. Deur, "Dynamic programming-based optimization of control variables of an extended range electric vehicle," SAE World Congress \& Exhibition 2013, 2013.

[6] B. Vural, A. R. Boynuegri, I. Nakir et al., "Fuel cell and ultra-capacitor hybridization: a prototype test bench based analysis of different energy management strategies for vehicular applications," International Journal of Hydrogen Energy, vol. 35, no. 20, pp. 11161-11171, 2010.

[7] P. García, J. P. Torreglosa, L. M. Fernández, and F. Jurado, "Viability study of a FC-battery-SC tramway controlled by equivalent consumption minimization strategy," International Journal of Hydrogen Energy, vol. 37, no. 11, pp. 9368-9382, 2012.

[8] B. Geng, J. K. Mills, and D. Sun, "Energy management control of microturbine-powered plug-in hybrid electric vehicles using the telemetry equivalent consumption minimization strategy," IEEE Transactions on Vehicular Technology, vol. 60, no. 9, pp. 4238-4248, 2011.

[9] A. Sciarretta, M. Back, and L. Guzzella, "Optimal control of parallel hybrid electric vehicles," IEEE Transactions on Control Systems Technology, vol. 12, no. 3, pp. 352-363, 2004.

[10] S. Barsali, C. Miulli, and A. Possenti, "A control strategy to minimize fuel consumption of series hybrid electric vehicles," IEEE Transactions on Energy Conversion, vol. 19, no. 1, pp. 187195, 2004.

[11] X. Wang, H. He, F. Sun, and J. Zhang, "Application study on the dynamic programming algorithm for energy management of plug-in hybrid electric vehicles," Energies, vol. 8, no. 4, pp. 3225-3244, 2015.

[12] D. Kum, H. Peng, and N. K. Bucknor, "Optimal energy and catalyst temperature management of plug-in hybrid electric vehicles for minimum fuel consumption and tail-pipe emissions," IEEE Transactions on Control Systems Technology, vol. 21, no. 1, pp. 14-26, 2013.

[13] C.-C. Lin, H. Peng, J. W. Grizzle, and J.-M. Kang, "Power management strategy for a parallel hybrid electric truck," IEEE Transactions on Control Systems Technology, vol. 11, no. 6, pp. 839-849, 2003.

[14] G. Rizzoni and S. Onori, "Energy management of hybrid electric vehicles: 15 years of development at the Ohio State University," Oil and Gas Science and Technology, vol. 70, no. 1, pp. 41-54, 2014.

[15] L. Xu, M. Ouyang, J. Li, F. Yang, L. Lu, and J. Hua, "Application of Pontryagin's Minimal Principle to the energy management strategy of plugin fuel cell electric vehicles," International Journal of Hydrogen Energy, vol. 38, no. 24, pp. 10104-10115, 2013.

[16] B. He, L. Lu, and J. Li, "Simulation research on energy management strategy of fuel cell hybrid electric vehicle," Journal of Highway and Transportation Research and Development, vol. 23, no. 1, pp. 151-154, 2006.

[17] X. Wu, C. Hu, and J. Chen, "Energy flow chart-based energy efficiency analysis of a range-extended electric bus," Mathematical Problems in Engineering, vol. 2014, Article ID 972139, 12 pages, 2014.

[18] Q. Gong, Y. Li, and Z. Peng, "Power management of plug-in hybrid electric vehicles using neural network based trip modeling," in Proceedings of the IEEE American Control Conference (ACC '09), pp. 4601-4606, St. Louis, Mo, USA, June 2009.

[19] N. J. Schouten, M. A. Salman, and N. A. Kheir, "Fuzzy logic control for parallel hybrid vehicles," IEEE Transactions on Control Systems Technology, vol. 10, no. 3, pp. 460-468, 2002.

[20] B.-C. Chen, Y.-Y. Wu, and H.-C. Tsai, "Design and analysis of power management strategy for range extended electric vehicle using dynamic programming," Applied Energy, vol. 113, pp. 1764-1774, 2014.

[21] Y. Zou, T. Liu, F. Sun, and H. Peng, "Comparative study of dynamic programming and Pontryagin's minimum principle on energy management for a parallel hybrid electric vehicle," Energies, vol. 6, no. 4, pp. 2305-2318, 2013.

[22] M. Montazeri-Gh and A. Fotouhi, "Traffic condition recognition using the k-means clustering method," Scientia Iranica, vol. 18, no. 4, pp. 930-937, 2011.

[23] C. Mansour and D. Clodic, "Optimized energy management control for the Toyota Hybrid System using dynamic programming on a predicted route with short computation time," International Journal of Automotive Technology, vol. 13, no. 2, pp. 309-324, 2012. 
[24] L. Xu, Powertrain System Modeling and Optimal Control of Fuel Cell Hybrid Electric Vehicle, Tsinghua University, Beijing, China, 2009.

[25] L. Tribioli, M. Barbieri, R. Capata, E. Sciubba, E. Jannelli, and G. Bella, "A real time energy management strategy for plug-in hybrid electric vehicles based on optimal control theory," Energy Procedia, vol. 45, pp. 949-958, 2014.

[26] L. Xu, M. Ouyang, J. Li, F. Yang, L. Lu, and J. Hua, "Optimal sizing of plug-in fuel cell electric vehicles using models of vehicle performance and system cost," Applied Energy, vol. 103, pp. 477-487, 2013.

[27] J. Hellgren, "Life cycle cost analysis of a car, a city bus and an intercity bus powertrain for year 2005 and 2020," Energy Policy, vol. 35, no. 1, pp. 39-49, 2007. 


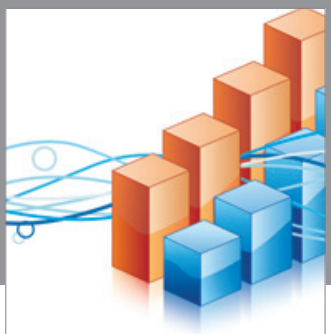

Advances in

Operations Research

vatem alat4

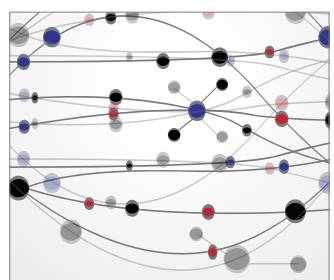

\section{The Scientific} World Journal
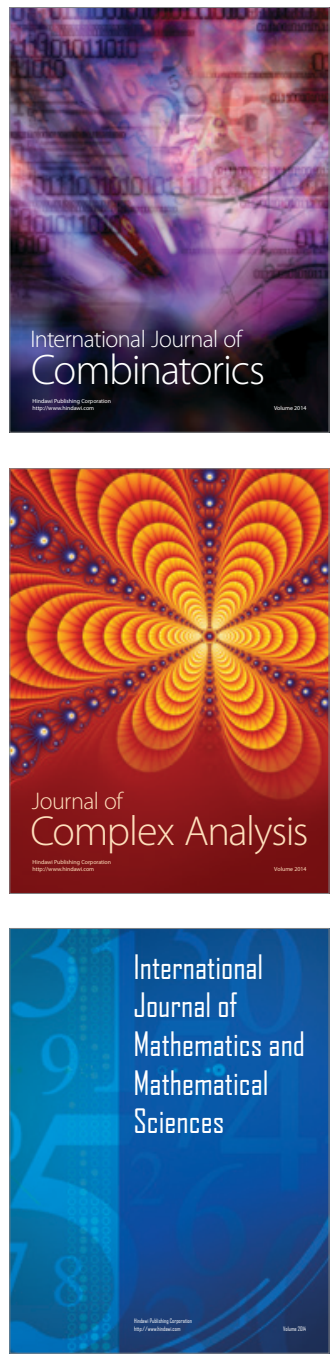
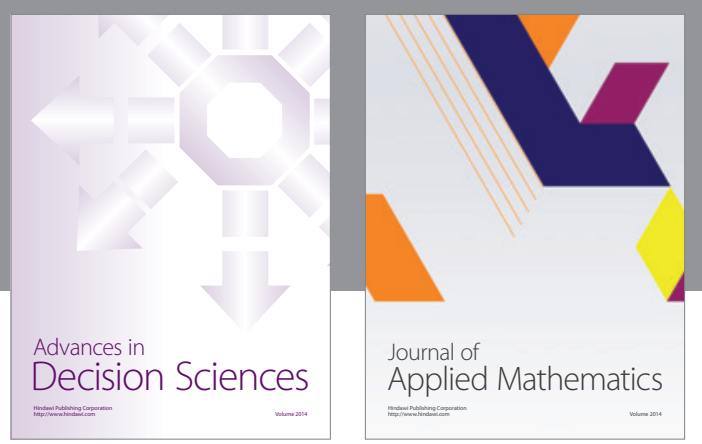

Algebra

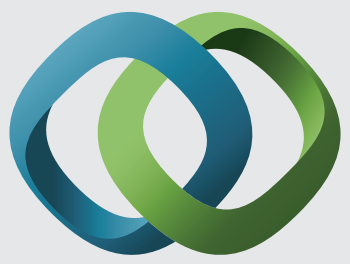

\section{Hindawi}

Submit your manuscripts at

http://www.hindawi.com
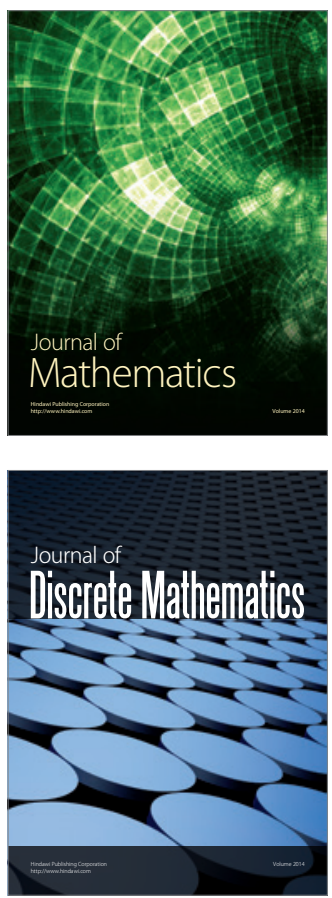

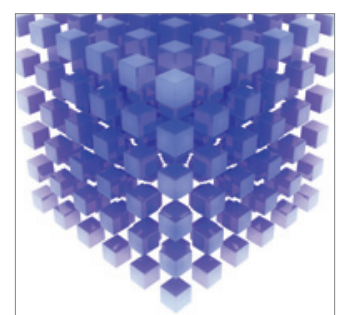

Mathematical Problems in Engineering
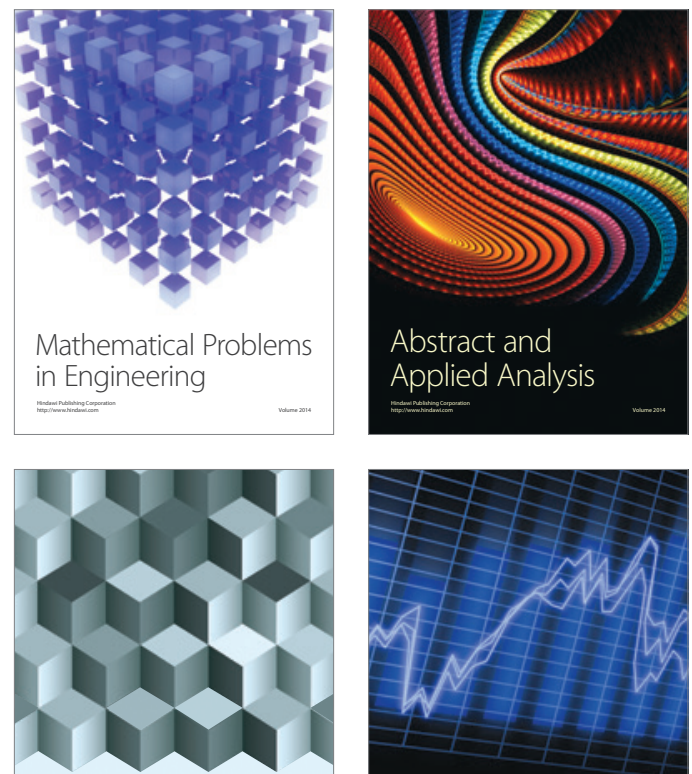

Journal of

Function Spaces

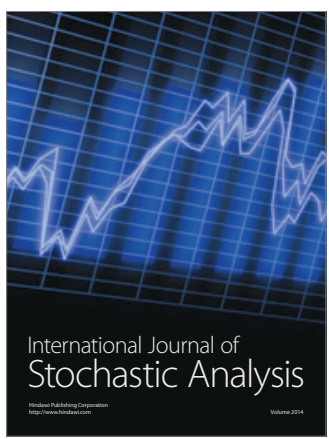

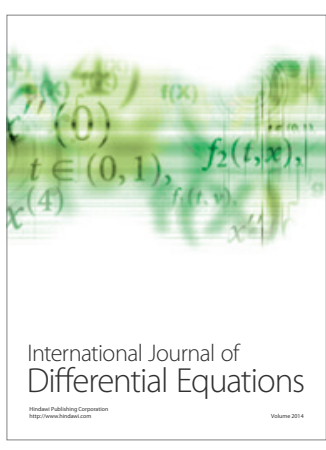
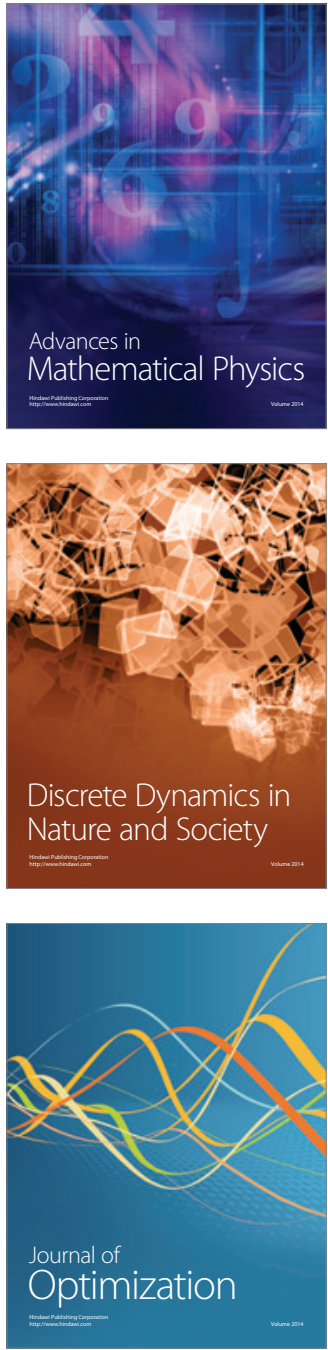